\title{
Enhancement of Solute Removal in a Hollow-Fiber Hemodialyzer by Mechanical Vibration
}

\author{
Jeong Chul Kim ${ }^{a-c}$ Francesco Garzotto ${ }^{a, b}$ Dinna N. Cruz ${ }^{a, b}$ Ching Yan Goh a, b, g \\ Federico Nalesso $^{a, b}$ Ji Hyun Kim ${ }^{d}$ Eungtaek Kang ${ }^{\mathrm{e}}$ Hee Chan Kim ${ }^{c, f}$ \\ Claudio Ronco ${ }^{\mathrm{a}, \mathrm{b}}$ \\ aDepartment of Nephrology, San Bortolo Hospital, and ' ${ }^{\mathrm{b}}$ International Renal Research Institute Vicenza, \\ Vicenza, Italy; ' Institute of Medical and Biological Engineering, Medical Research Center, Seoul National University, \\ ${ }^{\mathrm{d}}$ Interdisciplinary Program of Bioengineering Major, Seoul National University, e Department of Internal Medicine, \\ Chung-Ang University Hospital, and fDepartment of Biomedical Engineering, College of Medicine, Seoul National \\ University, Seoul, Korea; ${ }^{9}$ Nephrology Department, Selayang Hospital, Selangor, Malaysia
}

\section{Key Words}

Hemofiltration - Hemodiafiltration - Vibration - Mechanical stress - Concentration polarization • Membrane fouling • Diffusion · Convection · Adsorption

\begin{abstract}
Better solute clearance, particularly of middle-molecularweight solutes, has been associated with improved patient outcomes. However, blood-membrane interaction during dialysis results in the development of secondary mass transfer resistances on the dialyzer membrane surface. We discuss the potential effects of mechanical vibration on the diffusion, convection and adsorption of uremic solutes during dialysis. For sinusoidal and triangular vibratory motions, we conceptualized the hemodynamic changes inside the membrane and consequent effects on membrane morphology. Longitudinal vibration generates reverse flow by relative membrane motion, and transverse vibration generates a symmetric swirling flow inside the hollow fiber, which enhances wall shear stress and flow mixing. Moreover, the impulse induced by triangle wave vibration could provide
\end{abstract}

higher absorption capacity to middle-molecular-weight solutes. Mechanical vibration could enhance solute removal by minimizing membrane morphology changes resulting from blood-membrane interaction during hemodialysis. These effects of mechanical vibration can be helpful in extracorporeal blood purification therapies including continuous, portable and wearable systems.

Copyright $\odot 2011$ S. Karger AG, Basel

\section{Introduction}

In renal replacement therapy using an extracorporeal circuit, contact between blood and the artificial membrane, which has an enormous surface area, activates the coagulation pathway, complement cascades and immune system [1-3]. At the same time, when ultrafiltration occurs by transmembrane pressure gradient, hydraulic resistance of the membrane increases with treatment time due to concentration polarization and protein gel layer formation, which results in the reduction in dialysis efficiency [4-6]. In cross-filtration using hollow fiber mem-

\section{KARGER}

Fax +4161306 1234 E-Mail karger@karger.ch www.karger.com
(C) 2011 S. Karger AG, Basel

0253-5068/11/0314-0227\$38.00/0

Accessible online at:

www.karger.com/bpu
Claudio Ronco, MD

Department of Nephrology, San Bortolo Hospital

Viale Rodolfi 37

IT-36100 Vicenza (Italy)

Tel. +39 0444753 650, Fax +390444 753 973, E-Mail cronco@ goldnet.it 




Fig. 1. Development of concentration polarization and protein gel layer in the vicinity of a hollow-fiber membrane during the crossfiltration process. The solute concentration profile in the radial direction (right) and additional hydraulic resistance components in the membrane pore (bottom) are shown. $\mathrm{M}=$ Hollow-fiber membrane; $\mathrm{UF}=$ ultrafiltration; $C_{b}=$ solute concentration of bulk flow; $C_{p}=$ solute concentration at the polarized layer; $J_{1}=$ convective flux towards the membrane; $J_{2}=$ shear-induced back diffusion away from the membrane; $R_{g}=$ hydraulic resistance from the protein gel layer; $R_{m}=$ intrinsic membrane resistance; $R_{a d}=$ hydraulic resistance from protein adsorption into membrane pores; $R_{p p}=$ hydraulic resistance from pore plugging.

branes, the solute concentration in the vicinity of membrane surface is higher than that in bulk flow, developing in mass boundary layer (concentration polarization) which increases the resistance to ultrafiltration $[7,8]$. The protein gel layer is also developed between the membrane surface and the concentration polarization layer [9].

Recently, high-volume convective therapy has become popular worldwide, which is attributable to its ability to improve the clearance of middle molecular uremic toxins. On account of this, the issues related to secondary resistance layers in the dialysis membrane and their consequences are mounting. However, it is difficult to mimic the sieving profiles of the natural kidney due to the development of secondary resistance layers, leading to membrane selectivity impairment $[10,11]$. In convective therapy using a hollow-fiber dialyzer, it is very important to preserve the initial membrane morphology (effective surface area and pore distribution) by inhibiting the additional resistance layers caused by blood-membrane contact.
There have been many efforts to evaluate concentration polarization and a protein gel layer in low-Reynolds-flow membrane processes like hemodialysis. As a hydrodynamic approach, vortices induced by flow instability augmentation can be applied to preserve membrane performance in the cross-filtration process [12]. Mechanical vibration is one of the effective methods to induce flow instability and to control these secondary resistance layers. Vibration generates higher wall shear stress (WSS) at the membrane surface, which reduces mass boundary layer effects and protein gel layer thickness. Furthermore, optimal operating conditions can be obtained by adjustment of vibration amplitude and frequency according to the purpose of the application. Some previous studies in industrial fields reported that a vibrating membrane system could enhance the filtration efficiency by a factor of up to dozens of that in the static process $[13,14]$. In medical applications, enhanced shear stress at the membrane surface induced by a rotating filter system can improve plasma exchange efficiency and the ultrafiltration rate in hemofiltration $[15,16]$. This kind of dynamic shear-enhanced membrane system can be an alternative to avoid the limitations of static extracorporeal renal replacement therapy in middle molecular uremic toxin removal.

In this paper, we address the rationale for the application of mechanical vibration to an extracorporeal blood purification system to prevent the reduction of dialysis efficiency by blood-membrane contact. We briefly review the physics of concentration polarization and protein gel layer development at the membrane surface and suggest available mechanical vibration profiles in extracorporeal renal replacement therapy. The effects of these mechanical vibrations on hemodynamics in a hollow-fiber dialyzer are conceptualized to predict the solute removal during dialysis.

\section{Concentration Polarization and Protein Gel Layer in a Hollow-Fiber Membrane}

The pore size of hollow-fiber membranes used for hemodialysis ranges from 0.2 to $3.3 \mathrm{~nm}$, and the cutoff size of high-flux membranes is determined by the size of albumin (molecular weight 66,000) [17]. When uremic solutes are selectively removed from the blood through the dialysis membrane with various pore size distribution, concentration polarization and a protein gel layer can develop on the membrane surface (fig. 1). Although concentration polarization and the protein gel layer are physically different, they are related and influence each other. Concentra- 
tion polarization accelerates pore plugging, whereas a protein gel layer and blocked membrane pores influence the concentration polarization profile [18]. In terms of physics, concentration polarization is the mass boundary layer effect of an individual solute, while protein gel layer like cake formation is additional structural resistance [19]. In the membrane science field, Song and Elimelech [8] suggested a thermodynamic parameter, the so-called 'filtration number', to distinguish these two phenomena:

$$
N_{F}=\frac{4 \pi a_{p}^{3}}{3 k T} \Delta P
$$

where $N_{F}$ is the filtration number, $a_{p}$ is the particle radius, $k$ is the Boltzmann constant $\left(1.3803 \times 10^{-23} \mathrm{~J} / \mathrm{K}\right), T$ is the absolute temperature and $\Delta P$ is the pressure drop across the concentration polarization layer. If the filtration number was less than 15 , a protein gel layer did not develop on the membrane surface. In the ultrafiltration process of extracorporeal blood purification therapy, the range of the filtration number is from 0.1 to $10^{6}$, which is proportional to the third power of the radius of the particle. Thus, the filtration of middle molecular uremic toxins whose sieving coefficient is less than 1.0 can trigger the development of a protein gel layer during extracorporeal blood purification therapy.

\section{Concentration Polarization}

In a previous study, in the steady-state cross-filtration process, the ultrafiltration rate $\left(J_{f}\right)$ was expressed as a function of the bulk phase of plasma concentration $\left(C_{b}\right)$, shear rate at the membrane surface $(\gamma)$ and distance from the membrane inlet $(X)[20]$ :

$$
J_{f}=3.40 \times 10^{-5}\left[\frac{\gamma}{X}\right]^{1 / 3} \ln \frac{28.7}{C_{b}}
$$

Based on this equation, the ultrafiltration rate is proportional to the shear rate and decreases with protein concentration in the blood and distance from the inlet. In clinical practice, we can reduce the thickness of the concentration polarization layer by increasing the blood flow rate because WSS is proportional to the average velocity inside the membrane [4]. However, this approach could be impractical because there are limitations to achieving a high blood flow rate in clinical practice, related, for example, to the vascular access or hemodynamic status of the patient.

\section{Protein Gel Layer}

A protein gel layer develops between the membrane surface and the concentration polarization layer when the hydrodynamic condition (filtration number) reaches a critical value (fig. 1) [20]. This phenomenon also decreases the porosity of the membrane by adsorption of proteins and pore plugging. In this filtration environment, the hydraulic resistance of a membrane $(R)$ can be represented as

$$
R=R_{g}+R_{a d}+R_{p p}
$$

where $R_{g}$ is resistance from the gel layer, $R_{a d}$ is resistance from protein adsorption into pores and $R_{p p}$ is resistance from pore plugging [21]. Therefore, total membrane resistance $\left(R_{\text {total }}\right)$, taking into account both concentration polarization and the protein gel layer, can be expressed as

$$
R_{\text {total }}=R_{c p}+R_{g}+R_{m}+R_{a d}+R_{p p}
$$

where $R_{m}$ is the intrinsic membrane resistance and $R_{c p}$ is resistance from concentration polarization.

\section{Available Mechanical Vibration Profiles}

We can consider the application of mechanical vibration to extracorporeal blood purification to increase the shear rate at the membrane surface and to preserve membrane morphology during treatment. Mechanical vibration is feasible in clinical practice because it can provide high WSS enhancement with a simple mechanical mechanism in a limited space. There can be many profiles of mechanical vibration to inhibit the development of additional mass transfer resistances according to the direction and trajectory of motion. However, we focus on one-dimensional vibratory motion to simplify the relationship between mechanical vibration profiles and solute removal mechanisms. For one-dimensional longitudinal and transverse vibrations, we considered sinusoidal and triangular wave movement profiles for each vibration direction (fig. 2). Mathematically, position $x(t)$, velocity $v(t)$ and acceleration $a(t)$ for sinusoidal vibration are described as

$$
x(t)=A \sin (\omega t), v(t)=A \omega \cos (\omega t), a(t)=-A \omega^{2} \sin (\omega t)
$$

where $A$ is the amplitude of vibration and $\omega=2 \pi f$ ( $f$ is the frequency). In sinusoidal vibration, the amplitude of vibration increases by $\omega$ times from position to velocity, velocity to acceleration. In comparison, for triangle wave vibration, the velocity profile is a square wave and the acceleration increases to infinite at vertices of triangle waves. 


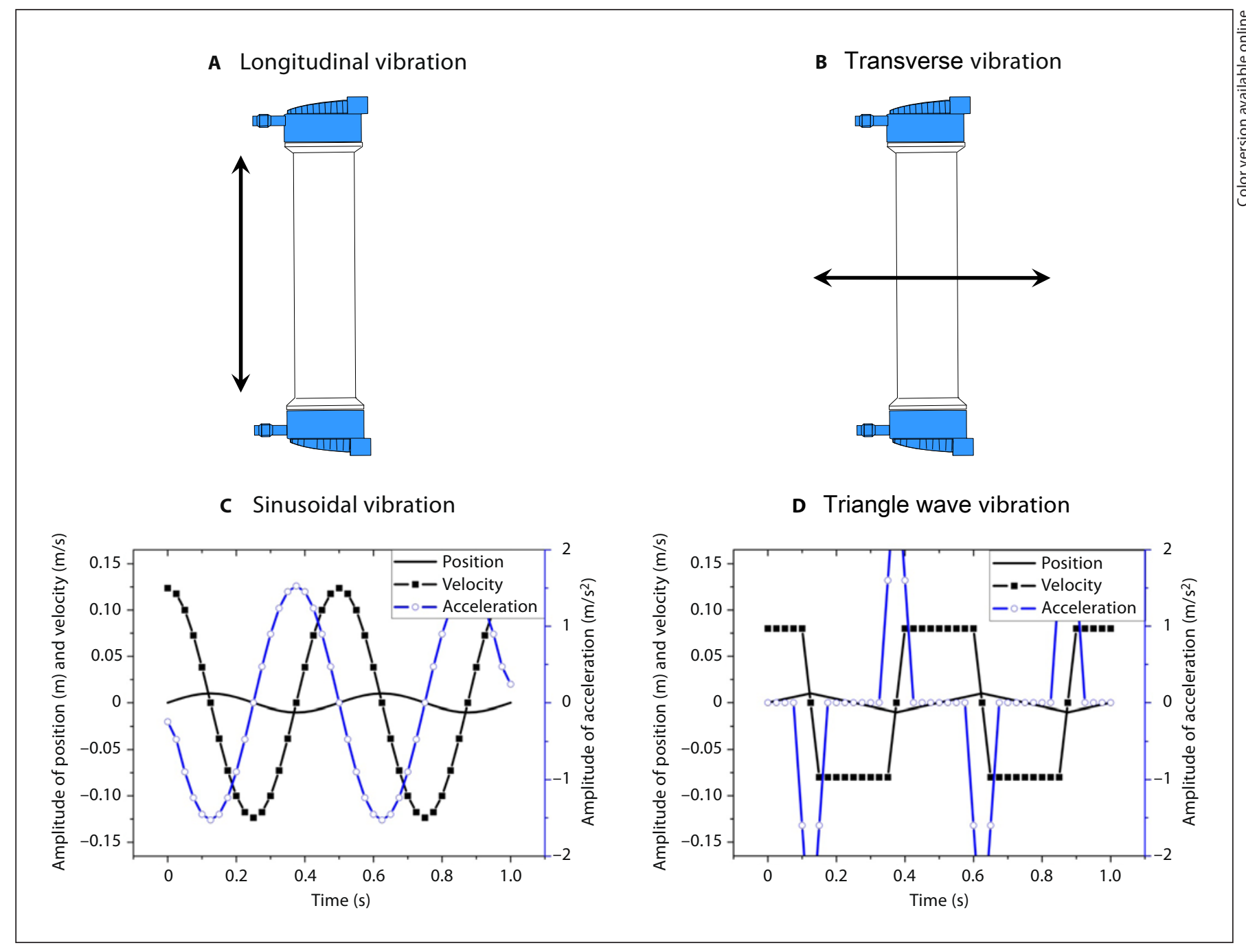

Fig. 2. A-D Mechanical vibration directions and position, velocity and acceleration according to vibration profiles when the vibration amplitude is $1 \mathrm{~cm}$ and the frequency is $2 \mathrm{~Hz}$. In $\mathbf{D}$, velocity and acceleration profiles are approximated and underestimated during the change of stroke direction for simple comparison with sinusoidal vibration.

\section{Longitudinal and Transverse Vibrations with \\ Sinusoidal Motion}

Theoretical and experimental studies on the effects of longitudinal vibration of a hollow fiber on membrane flux have been popular in the engineering field. During the forward stroke of vibration, when wall velocity is faster than the average velocity of the fluid, flow reversal occurs, which increases WSS at the membrane surface (fig. 3A). The backward stroke has the same effect as the forward one except for opposite flow direction [22] (fig. 3B). Therefore, we can expect a cleaning effect at the membrane surface from high WSS and alternatively changing flow reversal in a vibratory hollow-fiber system.

In a study on longitudinal vibration of a hollow fiber membrane with shear-thinning fluid like blood, the enhancement of WSS was initially linearly proportional to amplitude and frequency, but after certain points, the enhancement curves saturate as these vibration parameters further increased [14]. When one-dimensional transverse vibration is applied, symmetric swirling flow at the crosssection of a hollow fiber can be developed as shown in figure $3 \mathrm{C}$ and $\mathrm{D}$. In a previous study on pipe flow, the swirling or spiral flow developed by transverse vibration en- 
Fig. 3. Velocity profiles inside a single hollow fiber according to the vibration direction and stoke direction. A, B Longitudinal view of a hollow fiber. C, D Cross-sectional view of a hollow fiber.

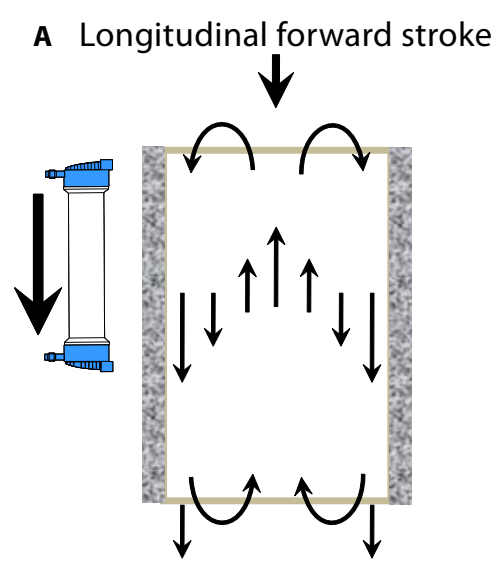

C Transverse right stroke

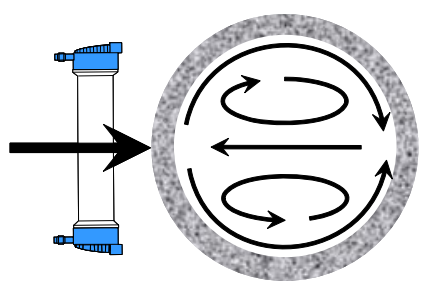

B Longitudinal backward stroke

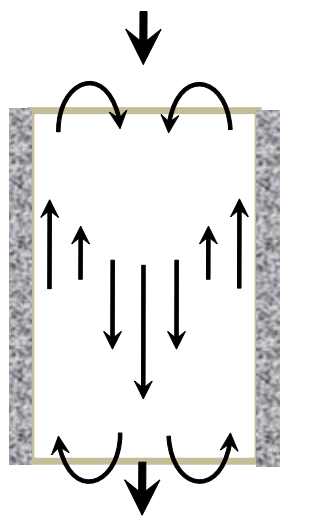

D Transverse left stroke

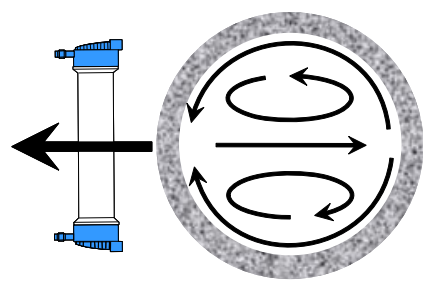

hanced radial temperature mixing [23]. In that study, it was also found that the amplitude to fiber diameter ratio had a linear relationship with the degree of radial mixing during transverse vibration. The same radial mixing enhancement was observed at the same amplitude of acceleration. Considering the physical similarity between heat transfer and mass transfer, we can apply the transverse vibration profile to control concentration polarization and protein gel layer formation in a hollow-fiber membrane.

\section{Longitudinal and Transverse Vibration Profiles with}

Triangle Wave Motion

Adsorption is one of the important mechanisms for middle molecular solute removal [24]. The materials and surface potential of hollow-fiber membranes are involved in solute removal by adsorption. For example, the primary mechanism of beta- 2 microglobulin removal is adsorption in sulfonated polyacrylonitrile and polymethylmethacrylate membranes compared to polysulfone membranes [25]. In these membranes, when low-molecular-weight proteins are absorbed into membrane pores, they cause partial or full pore blocking, which changes the membrane morphology with regard to pore size distribution and porosity.

Enhanced Solute Removal with Mechanical Vibration
Triangle wave vibration motion generates similar flow profiles inside a hollow fiber to those of sinusoidal vibration. Apart from WSS enhancement, triangular vibration also has the potential to improve adsorption. During the triangle wave vibration period (fig. 2D), although the amplitude of velocity ( $4 A f)$ is smaller than that of sinusoidal motion, there are two acceleration peaks when the stroke direction changes. At this moment, the impulse is transferred to the whole membrane module, which allows adsorbed particles to desorb from the blocked pore (fig. 1). Therefore, with triangular vibrations, we can improve the adsorption capacity of those hollow-fiber dialyzers in which the primary mechanism in middle molecule removal is adsorption. In terms of desorption, transverse vibration with a triangular profile might be more effective than a longitudinal one due to the impulse direction being parallel to the membrane pore.

\section{How to Apply Mechanical Vibration in Clinical \\ Practice}

In a preliminary study, we analyzed the effects of mechanical vibration on clearance of urea (molecular weight 60 ) and creatinine (molecular weight 113) during hemodialysis [26]. We developed an oscillating dialyzer holder

Blood Purif 2011;31:227-234 


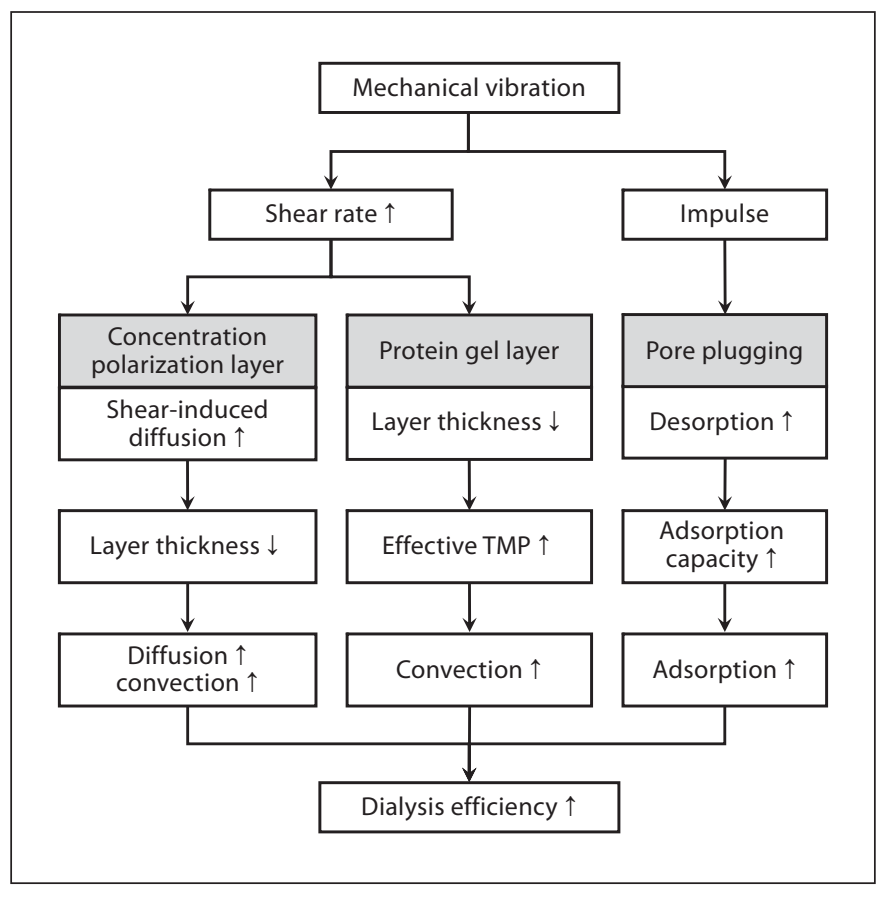

Fig. 4. Effects of mechanical vibration on hemodynamics and development of secondary layers inside the membrane, and their effects on the solute removal mechanism. TMP $=$ Transmembrane pressure.

using a crank-rocker mechanism, fixed the hemodialyzer to the rocker and applied mechanical vibration in clinical practice. In this 4-bar linkage, while the shorter side link revolves, the other one rocks (i.e. oscillates). In a stable hemodialysis patient, we performed a crossover study (without vibration and with vibration) during the hemodialysis sessions $\left(Q_{b}=350 \mathrm{ml} / \mathrm{min}, Q_{d}=500 \mathrm{ml} / \mathrm{min}\right.$, UF $=9.8 \mathrm{ml} / \mathrm{min}$ ). The angular velocity of the crank was 8.2 $\mathrm{rad} / \mathrm{s}$, and the amplitude and frequency of the rocker were $19^{\circ}$ and $1.3 \mathrm{~Hz}$, respectively. The solute clearance was improved by $9.8 \%$ for urea and $11.3 \%$ for creatinine, without significant damage to blood cells as measured by lactate dehydrogenase. This preliminary study suggested that solute clearance can be improved with appropriate mechanical vibration in clinical practice.

\section{Discussion}

In this study, we addressed the feasibility of employing mechanical vibration to preserve dialysis membrane performance during extracorporeal blood purification therapy. Vibration enhances relative fluid motion and increases WSS at the membrane surface in a limited user space $[27,28]$. A high wall shear rate induced by mechanical vibration inhibits the development of secondary mass transfer resistances like concentration polarization and protein gel layer. Additionally, triangular vibratory motion might enhance the desorption of proteins by instantaneous impulse. Therefore, mechanical vibration can be a good alternative method to improve diffusion, convection and adsorption during extracorporeal blood purification therapy (fig. 4).

The diffusion process in a hollow-fiber membrane is composed of Brownian motion and shear-induced diffusion. While small molecular solutes like urea and creatinine are controlled by Brownian motion, middle or macro-molecules are controlled by shear-induced diffusion [7]. Shear-induced diffusion influences the thickness of the concentration polarization layer; as shear-induced diffusion increases $\left(J_{2}\right.$ in fig. 1$)$, the thickness of the layer decreases due to the radial mixing. Shear-induced diffusion is proportional to both the wall shear rate and the square of the particle radius [29]. A previous study showed a decrease in solute clearance with molecular weight increase under zero net ultrafiltration conditions. This result partially explains the fact that middle molecular clearance is more influenced by concentration polarization layer $[5,11]$. Therefore, in a vibratory hollow-fiber system, enhanced lift force on solutes and shear-induced diffusion could improve middle-molecule clearance by controlling the thickness of the concentration polarization layer.

Concentration polarization and protein gel layer are directly related to effective transmembrane pressure in a hollow-fiber system. Transmembrane pressure is one of the important factors directly related to albumin loss in postdilution hemodiafiltration [30]. When secondary layers are developed at the membrane surface, the hemodiafiltration system requires a higher transmembrane pressure to deliver the targeted treatment dose. At the same time, membrane selectivity is impaired and this hydraulic environment leads to albumin loss during treatment. If we can maintain the solute and water permeability of the membrane using mechanical vibration, we don't have to use glucose infusion or gradual ultrafiltration control [31]. Therefore, mechanical vibration can be an alternative to improve middle-molecule clearance in treatments that utilize a high exchange volume with reduction of albumin loss.

Adsorption profiles can differ depending on the membrane material and surface potential. Negatively charged membranes are more likely to adsorb proteins with a positive charge and more likely to produce blood residues at 
the membrane surface. Using specific vibration profiles with high acceleration, we can generate an impulse on the membrane module and enhance desorption of solutes. However, when we apply mechanical vibration to an extracorporeal system, the risk of hemolysis should be taken into account. In longitudinal vibration, local high WSS can be observed due to high acceleration and a high area ratio in the orfice-structured region between the arterial port and hollow fibers. This hemodynamic circumstance could be one of the factors that increase blood cell damage at the arterial port of hollow-fiber hemodialyzers [32].

Besides the issue of solute clearance, we need to consider surface reaction at the interface of blood and dialysis membrane. The interaction of activated proteins adsorbed at the membrane surface might trigger several biological pathways, during which several protein reactions can be involved within a minute [33]. Mechanical vibration can modulate the flow pathway inside a hollow fiber, as shown in figure 3 . The changes in the flow pathway could increase net blood-membrane contact time and blood cell mixing by vortices in hollow fibers. Considering that higher WSS increases membrane adsorption of proteins [34], mechanical vibration may accelerate the blood-membrane reaction and could influence biocompatibility and long-term clinical outcomes. Recently, some studies quantified the amount of protein adsorbed into a membrane using time-of-flight secondary ion mass spectroscopy [35] and a proteomic approach [36]. They suggested the importance of adsorption control in membrane design for extracorporeal renal replacement therapy. Mechanical vibration can be useful for better solute clearance, but the operating conditions should be optimized in terms of blood-membrane surface reactions. Using these quantitative analysis methods, uremic solutes, proteins and amino acids patterns should be analyzed to confirm the feasibility of mechanical vibration in clinical practice.

Extracorporeal blood purification therapy using mechanical vibration could provide optimal treatment conditions for patients. Compared to steady-state conditions, with mechanical vibration we can maintain the membrane mass transfer performance and control the solute removal mechanism. These advantages may be helpful in continuous renal replacement therapy for the treatment of acute kidney injury. We can also apply this dynamic cross-filtration method to hollow-fiber membranes with a larger diameter and smaller surface area. This type of membrane is more stable from a hemodynamic point of view, and, ultimately, a miniaturized extracorporeal blood purification system such as a portable, wearable artificial kidney may be a suitable application target for mechanical vibration.

\section{Acknowledgements}

This work was supported by two National Research Foundation of Korea grants funded by the Korean Government (NRF2009-352-D00347 and MEST No. 2010-0002104).

\section{Disclosure Statement}

None of the authors have competing interests to declare.

\section{References}

1 Salzman EW: Thrombosis in artificial organs. Transplant Proc 1971;3:1491-1496.

-2 Craddock PR, Fehr J, Dalmasso AP, Brighan KL, Jacob HS: Hemodialysis leukopenia. Pulmonary vascular leukostasis resulting from complement activation by dialyzer cellophane membranes. J Clin Invest 1977;59: 879-888.

-3 Henderson LW, Koch KM, Dinarello CA, Shaldon S: Hemodialysis hypotension: the interleukin hypothesis. Blood Purif 1983;1: 3-8.

$\checkmark 4$ Ronco C, Ghezzi PM, Brendolan A, Crepaldi C, La Greca G: The haemodialysis system: basic mechanisms of water and solute transport in extracorporeal renal replacement therapies. Nephrol Dial Transplant 1998; 13(suppl 6):3-9.
5 Morti SM, Zydney AL: Protein-membrane interactions during hemodialysis: effects on solute transport. ASAIO J 1998;44:319-326.

-6 Huang Z, Gao D, Letteri JJ, Clark WR: Blood-membrane interactions during dialysis. Semin Dial 2009;22:623-628.

7 Bian R, Yamamoto K, Watanabe Y: The effect of shear rate on controlling the concentration polarization and membrane fouling. Desalination 2000;131:225-236.

8 Song L, Elimelech M: Theory of concentration polarization in crossflow filtration. J Chem Soc Faraday Trans 1995;91:33893398.
9 Chen JC, Li QL, Elimelech M: In situ monitoring techniques for concentration polarization and fouling phenomena in membrane filtration. Adv Colloid Interface Sci 2004;107:83-108.

10 Bowry SK: Nano-controlled membrane spinning technology: regulation of pore size, distribution and morphology of a new polysulfone dialysis membrane. Contrib Nephrol 2002;137:85-94.

11 Rockel A, Hertel J, Fiegel P, Abdelhamid S, Panitz N, Walb D: Permeability and secondary membrane formation of a high flux polysulfone hemofilter. Kidney Int 1986;30:429432.

12 Winzeler HB, Belfort G: Enhanced performance for pressure-driven membrane processes - the argument for fluid instabilities. J Memb Sci 1993;80:35-47. 
13 Genkin G, Waite TD, Fane AG, Chang S: The effect of vibration and coagulant addition on the filtration performance of submerged hollow fibre membranes. J Memb Sci 2006;281: 726-734.

14 Deshpande NS, Barigou M: Vibrational flow of non-Newtonian fluids. Chem Eng Sci 2001;56:3845-3853.

-15 Kaplan AA, Halley SE: Plasma exchange with a rotating filter. Kidney Int 1990;38: 160-166.

16 Okazaki M, Yoshida F: Ultrafiltration of blood: effect of hematocrit on ultrafiltration rate. Ann Biomed Eng 1976;4:138-150.

17 Uhlenbusch-Körwer I, Bonnie-Schorn E, Grassmann A, Vienken J: Understanding Membranes and Dialysers. Lengerich, Pabst Scientific, 2004.

-18 Hanhui Z, Jingjing Z, Dingti L, Xiaobin L: Reducing concentration polarization in hollow-fibre membranes. Membr Technol 2004; 5-9.

19 Kim ST: Characteristics of protein removal in hemodiafiltration. Contrib Nephrol 1994; 108:23-37.

20 Henderson LW: Biophysics of ultrafiltration and hemofiltration; in Jacobs C, Kjellstrand CM, Koch KM, Winchester JF (eds): Replacement of Renal Function by Dialysis, ed 4. Dordrecht, Kluwer Academic, 1996, pp 133-145.

21 Lenntech: Membrane fouling. http://www. lenntech.com/membrane-fouling.htm (accessed June 15, 2010).
22 Olayiwola B, Walzel P: Effects of in-phase oscillation of retentate and filtrate in crossflow filtration at low Reynolds number. J Memb Sci 2009;345:36-46.

-23 Eesa M, Barigou M: Enhancing radial temperature uniformity and boundary layer development in viscous Newtonian and nonNewtonian flow by transverse oscillations: a CFD study. Chem Eng Sci 2010;65:21992212.

-24 De Vriese AS, Colardyn FA, Philippe JJ, Vanholder RC, De Sutter JH, Lameire NH: Cytokine removal during continuous hemofiltration in septic patients. J Am Soc Nephrol 1999; 10:846-853.

-25 Clark WR, Gao DY: Low-molecular weight proteins in end-stage renal disease: potential toxicity and dialytic removal mechanisms. J Am Soc Nephrol 2002;13:S41-S47.

26 Garzotto F, Siragusa L, Cruz DN, Cenci N, Luison G, Parisotto F, et al: Enhancement of solute clearances by mechanical perturbation applied to hemodialyzers. ASN Renal Week, San Diego, Calif., 2009.

-27 Krantz WB, Bilodeau RR, Voorhees ME, Elgas RJ: Use of axial membrane vibrations to enhance mass transfer in a hollow tube oxygenator. J Memb Sci 1997;124:283-299.

28 Jaffrin MY: Dynamic shear-enhanced membrane filtration: a review of rotating disks, rotating membranes and vibrating systems. J Memb Sci 2008;324:7-25.
29 Eckstein EC, Bailey DG, Shapiro AH: Selfdiffusion of particles in shear flow of a suspension. J Fluid Mech 1977;79:191-208.

30 Santoro A, Canova C, Mancini E, Deppisch $\mathrm{R}$, Beck W: Protein loss in on-line hemofiltration. Blood Purif 2004;22:261-268.

31 Masakane I: Selection of dilutional method for on-line HDF, pre- or post-dilution. Blood Purif 2004;22(suppl 2):49-54.

>32 Kim JC, Kim JH, Sung J, Kim HC, Kang E, Lee SH, et al: Effects of arterial port design on blood flow distribution in hemodialyzers. Blood Purif 2009;28:260-267.

-33 Vroman L: Finding seconds count after contact with blood (and that is all I did). Colloids Surf B Biointerfaces 2008;62:1-4.

34 Valette P, Thomas M, Dejardin P: Adsorption of low molecular weight proteins to hemodialysis membranes: experimental results and simulations. Biomaterials 1999;20: 1621-1634

35 Aoyagi S, Hayama M, Hasegawa U, Sakai K, Tozu M, Hoshi T, et al: Estimation of protein adsorption on dialysis membrane by means of TOF-SIMS imaging. J Memb Sci 2004;236: 91-99.

36 Bonomini M, Pavone B, Sirolli V, Del Buono F, Di Cesare M, Del Boccio P, et al: Proteomics characterization of protein adsorption onto hemodialysis membranes. J Proteome Res 2006;5:2666-2674. 